\section{Custos para implantação e operação de serviço de saúde bucal na perspectiva do serviço e da sociedade}

\author{
The costs of implementing and operating an oral \\ health service from the perspective of the \\ service and society
}

\footnotetext{
${ }^{1}$ Clinica Integrada de Odontologia S.C., Sete Lagoas, Brasil.

Correspondência C. A. Ferreira Clinica Integrada de Odontologia S.C. Av. Prof. Mário Werneck 2618, apto.1500, Belo Horizonte, $M G$ 30750-180, Brasil. chrisaf2005@gmail.com
}

\begin{abstract}
The aim of this study was to assess the costs of setting up and maintaining dental care in the public sector. Costs were updated or depreciated according to the service's lifespan and were analyzed from the perspective of the service itself and society. According to the findings, for the service the total cost of setting up a dental care unit with seven rooms was BRL $\$ 860.643 .67$ in the first year, plus BRL\$545,419.23 for maintenance, and clinical dental care was the most expensive specialty. For society, the total cost was BRL\$990,065.06 (implementation) and BRL\$668,369.55 (maintenance), and the most expensive specialty was prevention (US\$1.00 = BRL\$1.62). Capital costs represented a small percentage of total costs for a dental care unit, but they need to be considered, since they can modify the results. Due to the high costs, preventive and promotional interventions should not be performed in the clinical setting, but should be replaced by broader and less expansive population-based interventions, since considerable sums need to be spent by the lowincome population to participate in free public programs.
\end{abstract}

Dental Health Services; Direct Service Costs; Dental Care
Christiane Alves Ferreira 1

Carlos Alfredo Loureiro 1

\section{Introdução}

Em se tratando de saúde, há uma tensão constante e natural entre o que serviço oferta e o que as pessoas gostariam que estivesse sendo ofertado. A base racional para a aplicação de recursos em saúde é fornecida por estudos de economia da saúde, os quais servem para orientar processos de decisão que visam obter máxima utilidade dos recursos investidos em uma situação de recursos finitos e escassos para satisfazer as necessidades de saúde das comunidades 1 .

Se não houvesse escassez de recursos, todas as ações poderiam ser implantadas e não haveria necessidade de estudos econômicos ou dos economistas, entretanto a realidade é diferente: os orçamentos são limitados, e quando se opta por aplicar os recursos em uma atividade, o uso desse recurso em outra atividade é negado (custo oportunidade). Instala-se, invariavelmente, uma competição pelos recursos financeiros e é difícil determinar qual área deveria ser sacrificada em prol da racionalização ${ }^{2}$.

A avaliação econômica visa identificar, quantificar e comparar os custos e as alternativas que são consideradas, e pode ser parcial (examina apenas custos) ou total (examina custos e conseqüências). Há também grande interesse em se determinar os custos do serviço da saúde para a sociedade, que inclui o custo do paciente 3 .

No Brasil, os serviços de saúde são gratuitos para a população, garantidos pela Constituição 
de 1988, e financiados pelo governo através de impostos e taxas. Ainda assim, serviços de saúde podem representar uma despesa importante para o paciente de baixa renda, particularmente em odontologia, em função da freqüência muitas vezes exigida pelos programas ofertados nos serviços.

Dessa forma, este estudo pretende avaliar economicamente o total de custos de implantação e operação da assistência odontológica no serviço público de saúde, sob o ponto de vista do serviço e da sociedade, tomando como exemplo um centro de especialidades de saúde bucal do Município de Sabará, Minas Gerais, Brasil.

\section{Métodos}

O delineamento utilizado foi o tipo pragmático, introduzido por Schwartz \& Lellouch ${ }^{4}$ que visa avaliar a eficiência de uma ou mais intervenções como custos e conseqüências nas condições reais de utilização, que prevalecem quando estas estão sendo utilizadas rotineiramente.

Foram coletados dados amostrais determinísticos e estocásticos. Para os dados amostrais determinísticos (edificação, mobiliário, equipamentos, instrumental, material médico hospitalar, manutenção e limpeza, água, telefone, luz, consultoria e alimentação) foi realizada a análise de sensibilidade. Para os dados amostrais estocásticos (salários, material de consumo e medicamentos odontológicos, transporte, tempo de tratamento, unidade básica clínica por especialidade, tratamento completado por especialidade) não foi necessário realizar análise de sensibilidade, porque foram obtidas a média e a variância.

A equipe clínica do Centro de Especialidades de Saúde Bucal (CESB) era constituída por 13 dentistas com carga horária de vinte horas semanais, sendo 11 clínicos gerais e dois especialistas: um endodontista e outro protesista e um higienista de quarenta horas. No setor administrativo eram: um gerente quarenta horas, cinco auxiliares de consultório dentário e dois agentes administrativos.

O centro avaliado contava com sete consultórios odontológicos equipados com aparelhos periféricos como: amalgamador, foto-polimerizador, ultra-som etc.; um setor de esterilização, uma recepção equipada com facilidades de informática, uma sala para higiene bucal, almoxarifado, sala de raios-X e uma sala de compressores.

O presente estudo avaliou os dados provenientes do CESB, que se encontrava em funcionamento na região desde julho de 2003. O período entre julho e dezembro do ano de 2003 foi utilizado para capacitação da equipe, treinamento e análises de dados e funcionamento dos sistemas operacionais. A partir de janeiro de $2004 \mathrm{o}$ CESB foi considerado plenamente operacionalizado, de acordo com os parâmetros previstos e, por esta razão, o ano de 2004 foi utilizado para a avaliação proposta neste estudo. Foram atendidos 4.848 pacientes eletivos e 2.978 pacientes de urgência, perfazendo um total de 7.826 pacientes de todas as idades, sendo que aproximadamente $40 \%$ deles foram atendidos em clínica geral. A política de acesso priorizava os casos mais graves, urgentes, ou com maior impacto dos problemas de saúde bucal na qualidade de vida. Esta política de acesso produziu um perfil de clientela mais complexo, devido ao acúmulo de necessidades resultante da alta incidência de doença bucal, à baixa condição econômica da população atendida, e por ter sido realizado, por algumas décadas, um atendimento público que incluía apenas a realização de exodontias e um atendimento precário ao escolar de até 14 anos de idade.

Alguns custos foram coletados na própria Secretaria Municipal de Saúde de Sabará, como salários, encargos, edificação e equipamentos. Outros custos tiveram que ser coletados diretamente na unidade avaliada, devido às dificuldades de obtenção de custos relativos a cada unidade odontológica.

Os instrumentos de rotina para mensurar a efetividade dos serviços foram os mesmos utilizados antes e validados por Loureiro et al. ${ }^{5} \mathrm{e}$ consistiam em uma unidade básica clínica e tratamento completado ou alta, considerados como conseqüências, longitudinalmente. Foram coletados dados de produtividade dos recursos humanos (RH), por especialidade, durante um período de nove meses para o cirurgião-dentista e 11 meses para o técnico de higiene dental que trabalhavam no CESB, no Município de Sabará, no ano de 2004. Cada unidade básica clínica correspondia a um período de tempo de vinte minutos. Para cada RH foi obtida a quantidade de turnos por dia e de dias úteis trabalhados por mês. O total de unidades básicas clínicas por dia foi então multiplicado pelo número de dias úteis, apurados mensalmente. A capacidade em unidade básica clínica foi dividida em unidade básica clínica efetiva (definida como aquela que resultou em atendimento a usuários) e ociosa (definida como aquela que não resultou em atendimento a pacientes). A unidade básica clínica efetiva foi subdividida em unidades básicas clínicas efetivas de pacientes que necessitavam de tratamento eletivo ou de tratamento de urgência. Paciente eletivo foi definido como qualquer tratamento realizado com agendamento antecipado nas especialidades: prevenção, clínica geral, endodon- 
tia ou prótese. As urgências representavam pacientes atendidos sem agendamento antecipado. A unidade básica clínica ociosa foi subdividida em não efetiva e não agendada. Unidade básica clínica não efetiva foi definida como aquela em que ocorreu agendamento do paciente, mas o atendimento não foi efetivado em função de falta do paciente, falta do $\mathrm{RH}$, problemas relativos à infra-estrutura do CESB, incluindo neste item desde falta de energia elétrica, água, material e medicamentos até a quebra de equipamentos.

Os dados de custo foram considerados em duas grandes áreas: custos do setor saúde e custos para a sociedade (paciente e família). Os custos do setor saúde foram desagrupados em custos de capital e custos de operação. Para custos de capital (edificação, equipamentos e periféricos) consideraram-se dois componentes para ajustamento dos valores: taxa de atualização e a depreciação.

Em estudos econômicos uma taxa de atualização anual é aplicada tanto sobre os custos como sobre as conseqüências, devido ao intervalo de tempo que decorre entre o início e o final do período avaliado, o que interfere no perfil de realização dos custos, bem como para obter as conseqüências da aplicação dos recursos. Ou seja, o custo operacional do CESB foi coletado mês a mês e adicionado para calcular o custo total durante o período avaliado. Entretanto, como decorreu um intervalo de tempo entre o primeiro e o último mês, é uma vantagem despender os recursos ao longo do ano, comparado a realizar todos os custos no primeiro mês de atividade da unidade. Esta situação é o que os economistas denominam "preferência no tempo", ou seja, na maioria das situações é preferível usufruir dos benefícios antes de arcar com os custos de um serviço ou bem. O valor da taxa de atualização é arbitrário e varia entre $3 \%$ e $6 \%$. Neste estudo foi adotada uma taxa de $5 \%$, por ser este o valor mais utilizado em estudos econômicos, o que favorece a comparação entre estudos diferentes, pelo menos nesta dimensão metodológica 6 .

O segundo ajuste aplicado se refere à taxa de depreciação ou amortização dos custos de capital. Esta taxa inclui nos custos o valor de bens como edificação e equipamentos odontológicos, de acordo com a vida útil do bem. Ou seja, se um consultório odontológico tem uma vida útil de dez anos, os custos da aquisição são amortizados em valores anuais por um período de dez anos. O custo obtido é denominado custo equivalente anual ou anuitização dos custos de capital. Como são necessários dois cálculos para realizar a atualização e a amortização, existem valores tabelados que combinam em um único fator qualquer taxa de atualização escolhida, com o tempo em anos para a vida útil do bem ${ }^{3}$. Para os itens de capital considerados foram adotados os seguintes períodos de vida útil: trinta anos (fator 15.3725) para edificação; dez anos (fator 7.7217) para equipamentos odontológico e mobiliário e; cinco anos (fator 4.3225) para periféricos, apoio e instrumental. Estes fatores correspondem a uma taxa de atualização de 5\%. Para custos operacionais (salários, material clínico e medicamentos, serviços de prótese, serviços de manutenção de equipamentos, água, telefonia, papelaria, impressos, consultoria, laudo, material médico hospitalar, material de limpeza e alimentação) foi aplicada apenas a taxa de atualização de 5\%, correspondente a um ano. Esta mesma taxa foi adotada para custos dos pacientes.

Os custos do paciente consideraram as despesas pagas diretamente pelo paciente ou sua família com o deslocamento até o CESB e o tempo gasto com o tratamento. Como referência para calcular o custo do paciente considerou-se o custo que um empregador teria para contratar uma hora extra de trabalho, tomando como referência a hora extra sobre o salário mínimo. O custo da hora extra foi calculado tomando como base o adicional de $100 \%$ sobre o salário mínimo adicionado por custos de FGTS (fundo de garantia do tempo de serviço), INSS (Instituto Nacional de Seguro Social), adicional de férias e 13o salário. Os valores obtidos foram então transformados em custo por unidade básica clínica de vinte minutos, o que resultou em $\mathrm{R} \$ 1,35 /$ unidade básica clínica, e este valor foi desagregado por especialidade. Para calcular o custo do tratamento foram obtidos os dados do tempo total despendido para realizar o tratamento para cada um dos 7.826 pacientes que receberam tratamento no CESB. O tempo de tratamento de cada paciente foi transformado em unidade básica clínica e multiplicado pelo custo da unidade básica clínica do paciente para obter o custo do tratamento na perspectiva do paciente, em cada especialidade.

Para cálculo do tempo de deslocamento cada paciente respondeu a um questionário aplicado como entrevista padronizada contendo três perguntas: meio de transporte utilizado, tempo de deslocamento e custo do transporte. Apenas dois meios de transporte foram identificados: transporte público e caminhada. O tempo médio de caminhada foi de vinte minutos e para calcular o custo da caminhada foi utilizado o mesmo método descrito para custo do tratamento. Para conhecer o custo do transporte público foi realizada uma média de preço das passagens. Neste estudo o custo da caminhada coincidiu com o custo médio das passagens e por esta razão, adotou-se um custo único para custo do deslocamento com valor médio igual a $\mathrm{R} \$ 1,83$ por 
unidade básica clínica. Para custo do paciente, o custo do deslocamento foi adicionado ao custo do tratamento perfazendo um valor médio igual a R\$ 3,18 por unidade básica clínica. O custo do paciente variava de acordo com o número de consultas que cada paciente necessitava receber, que interferia no número de deslocamentos, e com a duração do tratamento. Ou seja, tratamentos de mesma duração poderiam ter custos diferentes, dependendo do número de consultas. Tratamentos com pequena duração, mas que requeriam muitas consultas, resultavam em menor custo do tempo de tratamento, porém, com maior custo dos deslocamentos.

Para o compartilhamento dos custos de capital e de operação de departamentos diferentes do setor de odontologia (administração central, almoxarifado, licitação, transporte, eletricidade, material de uso comum etc.), adotou-se uma abordagem simplificada. Inicialmente foram identificados custos diretos relativos a cada unidade e em cada especialidade. O restante não diretamente atribuível foi alocado utilizando como direcionador de custos a quantidade de $\mathrm{RH}$ clínicos de cada unidade de atendimento, ou a quantidade de unidade básica clínica utilizada em cada especialidade.

$\mathrm{O}$ acesso aos dados deste estudo foi realizado com autorização do gestor municipal do Sistema Único de Saúde (SUS) e órgãos competentes da Prefeitura de Sabará e devidamente submetido ao Comitê de ética em Pesquisa do Centro de Pesquisas da São Leopoldo Mandic (número de protocolo 995) em 18 de março de 2003 e foi dispensado por este comitê, por não se tratar de pesquisas envolvendo seres humanos ou animais.

\section{Resultados}

A Tabela 1 apresenta o custo total anual do serviço e que não considera o custo do paciente, agrupado em custo de capital e de operação.

A mesma tabela mostra que o custo total em valores absolutos foi igual a $\mathrm{R} \$ 860.643,67$ e se refere ao custo do primeiro ano de funcionamento do CESB com sete consultórios o que representa

Tabela 1

Custo do serviço para o Centro de Especialidades de Saúde Bucal. Sabará, Minas Gerais, Brasil, 2004.

\begin{tabular}{|c|c|c|c|c|}
\hline \multirow[t]{2}{*}{ Custos } & \multirow[t]{2}{*}{ Parâmetro } & \multirow[t]{2}{*}{ Valores (R\$) } & \multicolumn{2}{|c|}{ Percentual } \\
\hline & & & Absoluto & Atualizado \\
\hline \multirow[t]{7}{*}{ Capital } & & & 38,31 & 7,29 \\
\hline & Custos & & & \\
\hline & Subtotal & $329.692,36$ & & \\
\hline & Atualizado e anuitizado & $39.751,32$ & & \\
\hline & Por unidade básica clínica & & & \\
\hline & Capacidade & 0,95 & & \\
\hline & Efetivada & 1,01 & & \\
\hline \multirow[t]{7}{*}{ Operação } & & & 61,69 & 92,71 \\
\hline & Custos & & & \\
\hline & Ano & $530.951,31$ & & \\
\hline & Atualizado & $505.667,91$ & & \\
\hline & Por unidade básica clínica & & & \\
\hline & Capacidade & 12,10 & & \\
\hline & Efetivada & 12,84 & & \\
\hline \multirow[t]{9}{*}{ Total do serviço } & & & 100,00 & 100,00 \\
\hline & Custos & & & \\
\hline & Total & $860.643,67$ & & \\
\hline & Por consultório & $122.949,10$ & & \\
\hline & Atualizado e anuitizado & $545.419,23$ & & \\
\hline & Por consultório & $77.917,03$ & & \\
\hline & Por unidade básica clínica & & & \\
\hline & Capacidade & 17,20 & & \\
\hline & Efetivada & 18,24 & & \\
\hline
\end{tabular}


um custo igual a $\mathrm{R} \$ 122.949,10$ por consultório. Em termos absolutos, custo de capital representa $38,31 \%$ dos custos e custos de operação $61,69 \%$. Considerando os custos anuitizados e atualizados o custo total do serviço foi igual a $\mathrm{R} \$ 545.419,23$ por ano, com custo de capital representando $7,29 \%$ dos custos e operação $92,71 \%$.

A Tabela 2 apresenta o custo total incluindo o custo do serviço e do paciente. Mostra que o total de custo absoluto encontrado foi igual a $\mathrm{R} \$ 990.065,06$, sendo que o custo do serviço representou $86,93 \%$ e do paciente $13,07 \%$. O total de custo anuitizado e atualizado foi igual a $\mathrm{R} \$$ $668.396,55$ com o custo do serviço representando $81,6 \%$ e do paciente $18,4 \%$. O custo para o atendimento eletivo por unidade básica clínica de vinte minutos foi igual a $R \$ 20,85$ e $R \$ 22,00$, por unidade capacidade e efetivada respectivamente, ou $\mathrm{R} \$ 62,55$ e $\mathrm{R} \$ 66,00$ por hora de atendimento. Para o atendimento de urgência o custo total da unidade básica clínica foi igual a $\mathrm{R} \$ 25,31$ ou
$\mathrm{R} \$ 26,53$ por unidade capacidade e efetivada, respectivamente, ou R \$75,93 e R \$ 79,59, por hora de atendimento.

A Tabela 3 apresenta o custo total do serviço e média por RH por mês, por tipo de atendimento, unidade básica clínica capacidade e efetivada e especialidade, sem considerar custo do paciente.

A Tabela 3 mostra que para o atendimento eletivo e unidade básica clínica efetivada, o maior custo total anuitizado e atualizado ocorreu com a especialidade clínica geral com $\mathrm{R} \$ 313.793,83$, seguida por prevenção com R $\$ 74.434,87$, prótese com R\$54.312,94, e endodontia com $\mathrm{R} \$ 32.013,14$. Quando comparado à média de custo por $\mathrm{RH}$, por mês, prótese apresentou o maior custo com R\$ 6.789,12 (IC95\%: 5.950,59-7.627,64) seguido por prevenção com R \$ 4.652,18 (IC95\%: $4.324,18-4.980,18)$, endodontia com $\mathrm{R} \$ 4.001,64$ (IC95\%: 3.505,09-4.498,19) e o menor custo foi com clínica geral com média R\$3.648,77 (IC95\%: $3.386,69-3.910,84)$.

Tabela 2

Total de custo incluindo custo do serviço e do paciente no Centro de Especialidades de Saúde Bucal. Sabará, Minas Gerais, Brasil, 2004

\begin{tabular}{|c|c|c|c|c|}
\hline \multirow[t]{2}{*}{ Custos } & \multirow[t]{2}{*}{ Parâmetro } & \multirow[t]{2}{*}{ Valores (R\$) } & \multicolumn{2}{|c|}{ Percentual } \\
\hline & & & Absoluto & Atualizado \\
\hline \multirow[t]{7}{*}{ Serviço } & & & 86,93 & 81,60 \\
\hline & Custos & & & \\
\hline & Total & $860.643,67$ & & \\
\hline & Atualizado e anuitizado & $545.419,23$ & & \\
\hline & Por unidade básica clínica & & & \\
\hline & Capacidade & 17,20 & & \\
\hline & Efetivada & 18,24 & & \\
\hline \multirow[t]{7}{*}{ Paciente } & & 13,07 & 18,40 & \\
\hline & Custos & & & \\
\hline & Ano & $129.421,39$ & & \\
\hline & Atualizado & $122.950,32$ & & \\
\hline & Por unidade básica clínica & & & \\
\hline & Capacidade & 3,18 & & \\
\hline & Efetivada & 3,39 & & \\
\hline \multicolumn{5}{|l|}{ Total } \\
\hline & Custo & & 100,00 & 100,00 \\
\hline & Total & $990.065,06$ & & \\
\hline & Atualizado e anuitizado & $668.369,55$ & & \\
\hline & Por unidade básica clínica & & & \\
\hline & Eletivo & & & \\
\hline & Capacidade & - & 20,85 & - \\
\hline & Efetivada & - & 22,00 & - \\
\hline & Urgência & & & - \\
\hline & Capacidade & - & 25,31 & \\
\hline & Efetivada & - & 26,53 & - \\
\hline
\end{tabular}


Custo total do serviço e média de custo por recursos humanos, mês anuitizado e atualizado por especialidade no Centro de Especialidades de Saúde Bucal. Sabará, Minas Gerais, Brasil, 2004.

\begin{tabular}{|c|c|c|c|c|c|c|c|c|c|c|c|c|}
\hline \multirow{4}{*}{$\begin{array}{l}\text { Atendi- } \\
\text { mento }\end{array}$} & \multirow{4}{*}{$\begin{array}{l}\text { Unidade } \\
\text { básica } \\
\text { clínica }\end{array}$} & \multirow{4}{*}{$\begin{array}{l}\text { Especiali- } \\
\text { dade }\end{array}$} & \multirow{2}{*}{\multicolumn{2}{|c|}{$\begin{array}{l}\text { Recursos } \\
\text { humanos }\end{array}$}} & \multicolumn{8}{|c|}{ Custo } \\
\hline & & & & & \multirow[t]{3}{*}{ Total } & \multirow[t]{3}{*}{$\%$} & \multirow{3}{*}{$\begin{array}{c}\text { Média por } \\
\text { recursos } \\
\text { humanos }\end{array}$} & \multirow{3}{*}{$\begin{array}{l}\text { Desvio- } \\
\text { padrão }\end{array}$} & \multicolumn{2}{|c|}{ IC95\% } & \multirow[t]{3}{*}{ Mínimo } & \multirow[t]{3}{*}{ Máximo } \\
\hline & & & $n$ & $\%$ & & & & & Inferior & Superior & & \\
\hline & & & & & & & & & & & & \\
\hline \multirow[t]{10}{*}{ Eletivo } & Capacidade & Prevenção & 1 & 13,56 & $68.676,23$ & 15,40 & $4.292,26$ & 643,92 & $3.949,14$ & $4.635,39$ & $2.718,69$ & $5.100,73$ \\
\hline & & Clínica geral & 11 & 72,88 & $297.507,18$ & 66,70 & $3.459,39$ & $1.168,65$ & $3.208,83$ & $3.709,94$ & 0,00 & $4.803,82$ \\
\hline & & Endodontia & 1 & 6,78 & $29.426,68$ & 6,60 & $3.678,33$ & 557,18 & $3.212,52$ & $4.144,15$ & $2.541,83$ & $4.307,88$ \\
\hline & & Prótese & 1 & 6,78 & $50.431,47$ & 11,31 & $6.303,93$ & $1.036,44$ & $5.437,45$ & $7.170,42$ & $3.989,74$ & $7.557,90$ \\
\hline & & Total & 14 & 100,00 & $446.041,55$ & 100,00 & $3.780,01$ & $1.294,58$ & $3.543,99$ & $4.016,03$ & 0,00 & $7.557,90$ \\
\hline & Efetivada & Prevenção & 1 & 13,56 & $74.434,87$ & 15,69 & $4.652,18$ & 615,55 & $4.324,18$ & $4.980,18$ & $3.007,25$ & $5.389,83$ \\
\hline & & Clínica geral & 11 & 72,88 & $313.793,83$ & 66,12 & $3.648,77$ & $1.222,36$ & $3.386,69$ & $3.910,84$ & 0,00 & $4.937,00$ \\
\hline & & Endodontia & 1 & 6,78 & $32.013,14$ & 6,75 & $4.001,64$ & 593,95 & $3.505,09$ & $4.498,19$ & $2.794,60$ & $4.591,07$ \\
\hline & & Prótese & 1 & 6,78 & $54.312,94$ & 11,45 & $6.789,12$ & $1.003,00$ & $5.950,59$ & $7.627,64$ & $4.556,05$ & $8.070,66$ \\
\hline & & Total & 14 & 100,00 & $474.554,78$ & 100,00 & $4.021,65$ & $1.376,83$ & $3.770,63$ & $4.272,67$ & 0,00 & $8.070,66$ \\
\hline \multirow[t]{8}{*}{ Urgência } & Capacidade & Clínica geral & 11 & 84,31 & $92.044,97$ & 92,62 & $1.070,29$ & $1.354,59$ & 779,87 & $1.360,72$ & 95,48 & $5.682,45$ \\
\hline & & Endodontia & 1 & 7,84 & $3.454,29$ & 3,48 & 431,79 & 175,44 & 285,12 & 578,46 & 222,04 & 726,24 \\
\hline & & Prótese & 1 & 7,84 & $3.878,42$ & 3,90 & 484,80 & 447,71 & 110,51 & 859,10 & 0,00 & $1.439,60$ \\
\hline & & Total & 13 & 100,00 & $99.377,68$ & 100,00 & 974,29 & $1.269,02$ & 725,03 & $1.223,55$ & 0,00 & $5.682,45$ \\
\hline & Efetivada & Clínica geral & 11 & 84,31 & $95.936,02$ & 92,41 & $1.115,54$ & $1.385,93$ & 818,39 & $1.412,68$ & 97,45 & $5.775,81$ \\
\hline & & Endodontia & 1 & 7,84 & $3.746,31$ & 3,61 & 468,29 & 187,94 & 311,17 & 625,41 & 249,02 & 798,46 \\
\hline & & Prótese & 1 & 7,84 & $4.134,27$ & 3,98 & 516,78 & 476,11 & 118,74 & 914,82 & 0,00 & $1.537,27$ \\
\hline & & Total & 13 & 100,00 & $103.816,60$ & 100,00 & $1.017,81$ & $1.298,70$ & 762,72 & $1.272,90$ & 0,00 & $5.775,81$ \\
\hline
\end{tabular}

A Tabela 4 apresenta o custo total do paciente por atendimento eletivo e urgência, por especialidade. Mostra que o custo total do paciente para o atendimento eletivo foi de R\$105.139,35, sendo que a especialidade de menor custo foi endodontia ( $\mathrm{R} \$ 4.462,92$ ou $4,24 \%$ ) e a de maior custo foi clínica geral (R\$ 58.365,45 ou 55,51\%). $\mathrm{O}$ atendimento de urgência teve um custo total de $\mathrm{R} \$ 17.810,97$, sendo que a especialidade de menor custo foi prótese ( $\mathrm{R} \$ 471,17$ ou $2,65 \%$ ) e a de maior custo foi clínica geral (R\$16.672,71 ou $93,61 \%)$.

\section{Discussão}

O custo total atualizado e anuitizado na perspectiva do serviço foi igual a $\mathrm{R} \$ 545.419,23$ para atender 7.826 pacientes em um ano. O custo de capital representou R \$ 39.751,32 (7,29\%) e o custo operacional foi igual a 505.667,91 (92,71\%). Comparando os tipos de custos, o custo operacional é maior que o custo de capital, especialmente quando são avaliados valores anuitizados. Como o custo de capital deve ser considerado de acordo com períodos relativamente longos de vida útil, representou um peso muito menor do custo total. Este resultado confirma que a inclusão de custos de capital não pode ser negligenciada em estudos econômicos, mas confirma também Normand ${ }^{7}$ que particularmente em relação ao custo de edificação, este representa uma participação menos importante no custo anual de serviços de saúde devido à sua diluição ao longo do tempo. O perfil encontrado em muitos municípios brasileiros indica freqüentemente uma quantidade adequada de RH e edificações e equipamentos em péssimas condições, utilizados muito além da vida útil. Esta situação pode comprometer a eficiência econômica na operação dos serviços que representa 12 vezes o custo de capital.

O custo de operação do serviço por cada consultório utilizado por oito horas apresentou um custo total igual a $\mathrm{R} \$ 77.917,03$ por ano. Este é o custo orçamentário que deve ser considerado para a expansão de um consultório odontológico no SUS, em uma situação semelhante à avaliada neste estudo.

Ao comparar a distribuição relativa dos itens de custo total na perspectiva do serviço, o principal item foi salário, que representou $66,36 \%$ do total, seguido por material odontológico com 
Custo total do paciente por tipo de atendimento e por especialidade no Centro de Especialidades de Saúde Bucal. Sabará, Minas Gerais, Brasil, 2004.

\begin{tabular}{|c|c|c|c|c|c|c|c|c|c|c|c|c|}
\hline \multirow{3}{*}{$\begin{array}{l}\text { Atendi- } \\
\text { mento }\end{array}$} & \multirow[t]{3}{*}{ Especialidade } & \multirow{2}{*}{\multicolumn{2}{|c|}{$\begin{array}{l}\text { Recursos } \\
\text { humanos }\end{array}$}} & \multirow[b]{3}{*}{ Total } & \multirow[b]{3}{*}{$\%$} & \multirow[b]{3}{*}{$\begin{array}{c}\text { Média por } \\
\text { recursos } \\
\text { humanos }\end{array}$} & \multirow{3}{*}{$\begin{array}{l}\text { Desvio- } \\
\text { padrão }\end{array}$} & \multirow{3}{*}{$\begin{array}{l}\text { Custo } \\
\text { Erro } \\
\text { padrão }\end{array}$} & \multirow{2}{*}{\multicolumn{2}{|c|}{ IC95\% }} & \multirow{3}{*}{ Mínimo } & \multirow{3}{*}{ Máximo } \\
\hline & & & & & & & & & & & & \\
\hline & & $\mathrm{n}$ & $\%$ & & & & & & Inferior & Superior & & \\
\hline \multirow[t]{5}{*}{ Eletivo } & Prevenção & 1 & 13,56 & $36.903,90$ & 35,10 & $2.306,49$ & 418,31 & 104,58 & $2.083,59$ & $2.529,40$ & $1.202,70$ & $2.785,20$ \\
\hline & Clínica geral & 1 & 72,88 & $58.365,45$ & 55,51 & 678,67 & 277,50 & 29,92 & 619,17 & 738,16 & 0,00 & $1.103,85$ \\
\hline & Endodontia & 1 & 6,78 & $4.462,92$ & 4,24 & 557,87 & 149,52 & 52,86 & 432,86 & 682,87 & 266,81 & 724,19 \\
\hline & Prótese & 1 & 6,78 & $5.407,08$ & 5,14 & 675,89 & 168,33 & 59,51 & 535,16 & 816,61 & 314,27 & 904,05 \\
\hline & Total & 14 & 100,00 & $105.139,35$ & 100,00 & 891,01 & 631,90 & 58,17 & 775,81 & $1.006,22$ & 0,00 & $2.785,20$ \\
\hline \multirow[t]{4}{*}{ Urgência } & Clínica geral & 11 & 84,31 & $16.672,71$ & 93,61 & 193,87 & 234,38 & 25,27 & 143,62 & 244,12 & 14,00 & 988,98 \\
\hline & Endodontia & 1 & 7,84 & 667,10 & 3,75 & 83,39 & 26,91 & 9,51 & 60,89 & 105,88 & 46,65 & 125,96 \\
\hline & Prótese & 1 & 7,84 & 471,17 & 2,65 & 58,90 & 57,83 & 20,45 & 10,55 & 107,25 & 0,00 & 186,60 \\
\hline & Total & 13 & 100,00 & $17.810,97$ & 100,00 & 174,62 & 220,34 & 21,82 & 131,34 & 217,90 & 0,00 & 988,98 \\
\hline
\end{tabular}

9,79\%, capital com 7,29\% e aquisição de serviços de prótese com $6,29 \%$. Este resultado quanto ao peso dos vencimentos difere de outros estudos. No estudo de Rosa \& Cauduro Neto 8 os autores encontraram que os vencimentos representavam $88 \%$ dos custos no setor público, mas o atendimento era restrito a crianças, e muitos itens de custo de capital não foram considerados. Utriainen \& Widström ${ }^{9}$ ao examinarem o custo de 34 centros de saúde na Finlândia encontraram que vencimentos representavam $76,4 \%$ dos custos e material odontológico 8,6\%, mas os autores não consideraram custo de capital. Morgan et al. 10, em estudo realizado na Austrália, encontraram que vencimentos representavam $71,7 \%$ e material odontológico $4,6 \%$ do total de custos, mas coletaram dados apenas para insumos de um programa preventivo destinado a crianças. Werner et al. 11 verificaram que salários representavam $91,67 \%$ e material odontológico $7,58 \%$ do custo total, mas os autores não consideraram custo de capital assim como muitos itens de custo operacional, além da avaliação ser restrita a um programa preventivo.

Os resultados obtidos neste estudo são de extrema importância para responder a questão sobre quanto custa implantar e operacionalizar serviços odontológicos no setor público, informação até então inexistente como dado publicado no Brasil. Os resultados de um estudo publicado por Rosa \& Cauduro Neto ${ }^{8}$, mesmo quando ajustados para a inflação no período, não são comparáveis devido a importantes diferenças metodológicas e conceituais, especialmente quanto aos conceitos de custos fixos e variáveis, diretos e indiretos, a não consideração de custos de capital, não utilização de taxa de atualização e aos autores não terem realizado análise de sensibilidade.

A comparação entre modalidades de atendimento indicou que $82,05 \%$ do custo total foram gastos no atendimento eletivo e $17,95 \%$ em urgência. No atendimento eletivo, aproximadamente dois terços dos custos $(66,12 \%)$ foram gastos com clínica geral, seguido por prevenção (15,69\%), prótese $(11,45 \%)$ e endodontia $(6,75 \%)$. Estes valores são importantes do ponto de vista da provisão orçamentária dos municípios. O fato de prevenção representar o segundo maior orçamento para atendimento eletivo reflete o atual modelo de saúde adotado no SUS que é predominantemente biomédico.

A média de custo por RH em cada especialidade fornece uma informação útil para se decidir sobre o peso que representará em termos orçamentários a implantação de um novo serviço ou a ampliação de um já existente. Nesta análise, prótese representou o maior custo total médio por RH, com diferença significante para todas as outras especialidades, seguida por prevenção, com custo maior quando comparado à endodontia, mas sem diferença significante comparada à clínica geral. Endodontia apresentou custo total médio por RH maior e com diferença significante comparada à clínica geral.

Este resultado confronta algumas crenças amplamente enraizadas no setor público em saúde bucal de que o atendimento eletivo em especialidades apresenta alto custo e não deve ser priorizado e que, ao contrário, atendimento pre- 
ventivo deve ser priorizado por ser de baixo custo. De acordo com os resultados obtidos, em nível de provisão orçamentária dos municípios, a decisão por implantar atendimento especializado, pelo menos no que se refere ao atendimento eletivo em endodontia, apresentou custo semelhante ao atendimento preventivo. Este resultado concorda com outros autores que avaliaram o custo de tratamento especializado. Järvinen \& Widström 12 ao avaliarem o custo do tratamento ortodôntico na Finlândia, uma especialidade considerada de alto custo, consideraram ser perfeitamente aceitável para o atendimento público.

Com relação ao custo da prevenção, os resultados do presente estudo corroboram os achados de Klock 13 que demonstraram que implantar este tipo de serviço, quando realizado na cadeira odontológica, mesmo se executado por um técnico de higiene dental, apresenta um custo relativamente alto porque demanda uso intensivo de $\mathrm{RH}$, o principal item de custo dos serviços. Duas revisões sistemáticas da literatura sobre a vantagem econômica em implantar programas de prevenção de cárie dentária realizados no ambiente clínico concluíram que os estudos originais revisados sobre avaliação econômica da prevenção não provêm suporte que indique existir valor econômico na implantação de programas de prevenção de cárie realizados em ambiente clínico 14,15. A conseqüência prática a ser considerada em uma análise de decisão é que, tomando como referência o custo por $\mathrm{RH}$, implantar um serviço preventivo em ambiente clínico na rede pública nas condições avaliadas neste estudo apresenta um custo semelhante ao de implantar um serviço especializado como endodontia. Do ponto de vista da priorização de serviços públicos, a discussão que este resultado suscita é se é eticamente correto rejeitar o princípio da solidariedade que obriga profissionais de saúde a priorizar aqueles que mais sofrem com a presença da doença instalada, em favor da prevenção de futuros problemas de saúde cuja efetividade e custo efetividade não são adequados. O mesmo tipo de discussão foi levantado por Schwartz 16 em seu estudo que analisou o custo efetividade da modalidade preventiva e sugeriu que se os profissionais de saúde querem destinar recursos para as medidas preventivas, é necessário averiguar se a sociedade está preparada para sacrificar o atendimento de problemas mais urgentes e mais graves e esperar o intervalo de tempo necessário para obter os efeitos dessas medidas preventivas.

Custos do paciente são pouco estudados em Odontologia. Oscarson et al. 17 ao compararem quatro protocolos concluíram que o custo do paciente representava entre $17,76 \%$ a $43,34 \%$ do custo total e tinha um alto impacto para crianças e adolescentes em programas preventivos, variando em até cinco vezes, de acordo com o protocolo utilizado entre uma a quatro visitas anuais. Estes valores são relevantes quando comparados a tratamentos de outras doenças. Costa et al. 18 avaliaram os custos do tratamento ambulatorial no serviço público para tuberculose na cidade de Salvador, Bahia, Brasil, e encontraram que estes correspondiam a 33\% do total. Entretanto, os autores consideraram as perdas de renda do doente por incapacitação temporária e do acompanhante, que representaram aproximadamente metade dos custos do paciente.

Comparando as perspectivas do serviço e da sociedade as conclusões são diferentes. Na perspectiva do serviço, o custo total médio por RH para prevenção é maior quando comparado à endodontia e clínica geral, mas a diferença não é significante para endodontia. Na perspectiva da sociedade, a diferença entre prevenção e endodontia é significante. Este resultado indica que o sacrifício imposto aos pacientes no serviço avaliado para participar de atividades preventivas realizadas na cadeira odontológica é maior em prevenção que nos outros atendimentos eletivos comparados. Neste estudo esta diferença se deveu ao fato da prevenção apresentar o maior custo relativo com transporte em comparação ao custo com o tempo de tratamento devido à repetição de consultas de curta duração.

Estes resultados confirmam a importância de realizar análises econômicas na perspectiva da sociedade, o que inclui o custo do paciente, pois os resultados obtidos demonstraram valores consideráveis que são desembolsados pela população para participar de programas públicos gratuitos, além disto, a inclusão destes custos modifica as conclusões do estudo. Ou seja, os resultados mostram claramente que programas gratuitos não significam programas sem custo para o paciente e a sua família. A utilidade prática deste resultado é que o custo do paciente pode implicar um impacto negativo na adesão da população de baixa renda a protocolos curativos ou preventivos que exigem muito tempo ou requerem muitas e repetidas visitas para se completar. Os resultados indicam que, ao contrário da tradição de ofertar consultas odontológicas de meia hora na maioria dos serviços públicos, o aumento do tempo de consulta para reduzir o número de visitas reduz o custo do paciente em razão do menor custo do transporte comparado ao do tempo de tratamento para a população de baixa renda.

É importante ressaltar que os resultados deste estudo se basearam em um contexto particular, relativos à população que utiliza os serviços do 
SUS, em um único centro odontológico, portanto os mesmos devem ser avaliados com cautela e repetidos em situações diferentes.

\section{Conclusão}

Os resultados deste estudo indicam que custos de capital representam uma parcela pouco representativa dos custos de um centro odontológico, entretanto, devem ser considerados nos estudos econômicos, pois modificam os resultados. Considerando o valor equivalente anual dos itens de capital, não existe racionalidade econômica para justificar o pouco caso atribuído a estes itens no SUS, em muitos municípios brasileiros, pois a deterioração destes itens pode comprometer a eficiência operacional que representa a grande parcela dos custos de um serviço odontológico.

Na perspectiva dos serviços, salários e vencimentos representam dois terços dos custos, enquanto material odontológico e serviços de prótese representam apenas um sexto dos custos. Estes dados sugerem que falhas de abastecimento de suprimentos, fato comum em muitos serviços odontológicos do SUS, podem comprometer a eficiência dos $\mathrm{RH}$, representando um alto custo da ineficiência do serviço.

\section{Resumo}

O objetivo deste estudo foi avaliar custos de implantação e manutenção da assistência odontológica no setor público. Os custos foram atualizados/depreciados de acordo com a vida útil e considerados na perspectiva do serviço e da sociedade. Os resultados mostraram que, para o serviço, o custo total de implantação de uma unidade odontológica com sete consultórios foi de $R \$ 860.643,67$ no primeiro ano e $R \$ 545.419,23$ para manutenção, sendo clínica geral a especialidade mais cara. Para a sociedade, o custo total foi de $R \$$ 990.065,06 (implantação) e $R \$ 668.369,55$ (manutenção) e a especialidade mais cara foi prevenção. Custos de capital representaram um pequeno percentual dos custos de uma unidade odontológica, entretanto, deveriam ser considerados, pois podem modificar os resultados. Devido ao alto custo, intervenções preventivo-promocionais realizadas no ambiente clínico não deveriam ser recomendadas, devendo ser substituídas por ações populacionais amplas e de menor custo, uma vez que valores consideráveis necessitam ser desembolsados pela população de baixa renda para participar de programas públicos gratuitos.

Serviços de Saúde Bucal; Custos Diretos de Serviços; Assistência Odontológica
Intervenções preventivo-promocionais realizadas por profissional de saúde em ambiente clínico representaram o segundo maior custo anual, ultrapassando os custos de especialidades como prótese e endodontia. Estes resultados sugerem que estas intervenções não deveriam ser recomendadas quando realizadas no ambiente clínico, devendo ser substituídas por ações populacionais amplas de menor custo. Este resultado confronta algumas crenças amplamente enraizadas no setor público, de que o atendimento eletivo em especialidades apresenta alto custo e não deve ser priorizado, e de que, ao contrário, atendimento preventivo deve ser priorizado por ser de baixo custo.

A consideração dos custos dos pacientes para obter tratamento no SUS altera significativamente os resultados da análise e, por esta razão não devem ser omitidos em estudos de custos. Os resultados indicaram que o sacrifício imposto aos pacientes no serviço avaliado para participar de atividades clínicas realizadas na cadeira odontológica é maior em prevenção que nos outros atendimentos eletivos comparados. A razão é que estas intervenções demandam menores intervalos de tratamento, o que implica mais consultas e maior custo de transporte.

\section{Colaboradores}

C. A. Ferreira contribuiu com o planejamento e a elaboração do projeto de pesquisa, a coleta de dados, a revisão da literatura, a análise de custos e redação do artigo. C. A. Loureiro colaborou com o planejamento e coordenação da pesquisa, com a redação do artigo e revisão do texto final. 


\section{Referências}

1. Donaldson C. Economic evaluation in dentistry: an ethical imperative? Dent Update 1998; 25:260-4.

2. White BA. The costs and consequences of neglected medically necessary oral care. Spec Care Dentist 1995; 15:180-6.

3. Drummond MF, O'Brien BJ, Stoddart GI, Torrance GW. Methods for the economic evaluation of health care programs. 2nd Ed. New York: Oxford University Press; 1997.

4. Schwartz D, Lellouch J. Explanatory and pragmatic attitudes in therapeutical trials. J Chronic Dis 1967; 20:637-48.

5. Loureiro CA, Araujo VE, Lopes MIP, Ayres JR. Pesquisa de avaliação da utilização de um método sistemático de mudança em um serviço de saúde bucal - BEPREM, MG. UFES Rev Odontol 2001; 3:68-78.

6. Johnston K, Buxton MJ, Jones DR, Fitzpatrick R. Assessing the costs of healthcare technologies in clinical trials. Health Technol Assess 1999; 3:1-76.

7. Normand C. Ten popular health economic fallacies. J Public Health Med 1998; 20:129-32.

8. Rosa AGF, Cauduro Neto R. Custos em odontologia; análise dos custos de um serviço odontológico do setor público. RGO (Porto Alegre) 1985; 33:242-3.

9. Ultriainen P, Widström E. Economic aspects of dental care in Finnish health centers. Community Dent Oral Epidemiol 1990; 18:235-8.

10. Morgan MV, Crowley SJ, Wright C. Economic evaluation of a pit and fissure dental sealant and fluoride mothrinsing program in two nonfluoridated regions of Vitoria, Australia. J Public Health Dent 1998; 58:19-27.
11. Werner CW, Pereira AC, Eklund SA. Cost-effectiveness study of a school-based sealant program. J Dent Child 2000; 67:93-7.

12. Järvinen S, Widström E. Determinants of costs of orthodontic treatment in the Finnish public health service. Swed Dent J 2002; 26:41-9.

13. Klock B. Economic aspects of a caries preventive program. Community Dent Oral Epidemiol 1980; 8:97-102.

14. Källestål C, Norlund A, Söder B, Nordenram G, Dahlgren H, Petersson LG, et al. Economic evaluation of dental caries prevention: a systematic review. Acta Odontol Scand 2003; 61:341-6.

15. Ferreira CA, Loureiro CA. Economia em saúde com foco em saúde bucal: revisão de literatura. Epidemiol Serv Saúde 2006; 15:55-65.

16. Schwarz E. Is caries prevention cost-effective? Does anybody care? Acta Odontol Scand 1998; 56:187-92.

17. Oscarson N, Kallestal C, Fjelddahl A, Lindholm L. Cost-effectiveness of different caries preventive measures in a high-risk population of Swedish adolescents. Community Dent Oral Epidemiol 2003; 31:169-78.

18. Costa JG, Santos AC, Rodrigues LC, Barreto ML, Roberts JA. Tuberculose em Salvador: custos para o sistema de saúde e para as famílias. Rev Saúde Pública 2005; 39:122-8.

Recebido em 16/Ago/2006

Versão final reapresentada em 07/Fev/2008 Aprovado em 28/Fev/2008 